\title{
Clarifying the Origin of Biological Abnormalities in PTSD Through the Study of Identical Twins Discordant for Combat Exposure
}

\section{Citation}

Pitman RK, Gilbertson MW, Gurvits TV, May FS, Lasko NB, Metzger LJ, Shenton ME, Yehuda R, Orr SP. 2006. Clarifying the origin of biological abnormalities in PTSD through the study of identical twins discordant for combat exposure. Ann N Y Acad Sci. 1071: 242-54. doi:10.1196/ annals.1364.019

\section{Published Version}

doi:10.1196/annals.1364.019

\section{Permanent link}

http://nrs.harvard.edu/urn-3:HUL.InstRepos:28566282

\section{Terms of Use}

This article was downloaded from Harvard University's DASH repository, and is made available under the terms and conditions applicable to Other Posted Material, as set forth at http:// nrs.harvard.edu/urn-3:HUL.InstRepos:dash.current.terms-of-use\#LAA

\section{Share Your Story}

The Harvard community has made this article openly available.

Please share how this access benefits you. Submit a story.

Accessibility 


\title{
Clarifying the Origin of Biological Abnormalities in PTSD Through the Study of Identical Twins Discordant for Combat Exposure
}

\author{
Roger K. Pitman ${ }^{a, b}$, Mark W. Gilbertson ${ }^{b, c}$, Tamara V. Gurvits ${ }^{b, c}$, Flavia S. May ${ }^{a}$, Natasha B. \\ Lasko a,b,c, Linda J. Metzgera,b,c, Martha E. Shenton ${ }^{b, d}$, Rachel Yehudae, and Scott P. \\ Orra,b,c for the Harvard/VA PTSD Twin Study Investigators \\ aDepartment of Psychiatry, Massachusetts General Hospital, Boston, Massachusetts, USA \\ bDepartment of Psychiatry, Harvard Medical School, Boston, Massachusetts, USA \\ 'Research Service, VA Medical Center, Manchester, New Hampshire, USA \\ dNeuroimaging Laboratory, Department of Psychiatry, and Surgical Planning Laboratory, MRI \\ Division, Department of Radiology, Brigham and Women's Hospital, Boston, Massachusetts, USA \\ ePsychiatry Service, James J. Peters VA Medical Center, Bronx, New York 10468, and Department \\ of Psychiatry, Mt. Sinai School of Medicine, New York 10029, USA
}

\begin{abstract}
A biological abnormality found to be associated with post-traumatic stress disorder (PTSD) may be, among other things, a pre-trauma vulnerability factor, that is, it may have been present prior to the event's occurrence and increased the individual's likelihood of developing PTSD upon traumatic exposure. Alternately, it may be an acquired PTSD sign, that is, it may have developed after the traumatic exposure, along with the PTSD. We have studied pairs of Vietnam combat veterans and their noncombat-exposed, identical twins in an effort to resolve these competing origins. Combat veterans were diagnosed as current PTSD or non-PTSD (i.e., never had). Average heart rate responses (HRRs) to a series of sudden, loud-tone presentations were larger in Vietnam combat veteran twins with PTSD, but these larger responses were not shared by their noncombat-exposed cotwins, whose responses were similar to those of the non-PTSD combat veterans and their noncombat-exposed cotwins. These results suggest that larger HRRs to sudden, loud tones represent an acquired sign of PTSD. In contrast, increased neurological soft signs (NSSs), diminished hippocampal volume, and presence of abnormal cavum septum pellucidum (CSP) were found in Vietnam combat veteran twins with PTSD and their "high-risk," unexposed cotwins compared to Vietnam combat veteran twins without PTSD and their "low-risk," unexposed cotwins. These results support the conclusion that the latter abnormalities represent antecedent, familial vulnerability factors for developing chronic PTSD upon exposure to a traumatic event.
\end{abstract}

\section{Keywords}

stress disorders; posttraumatic; twins; monozygotic; startle response; neurological examination; magnetic resonance imaging; hippocampus; septum pellucidum 


\section{INTRODUCTION}

Over the past two decades, posttraumatic stress disorder (PTSD) has been characterized by a number of biological abnormalities. Most of this research has been cross-sectional, that is, correlational, in nature. It is tempting to conclude that because PTSD is recognized as being caused by a psychologically traumatic environmental event, ${ }^{1}$ any abnormalities found in PTSD patients must have been caused by that event. However, this is not necessarily the case. Correlation does not prove causality. Indeed, there are at least five different possible explanations for an observed association between a given biological abnormality and PTSD. First, an abnormality may be an antecedent risk factor for exposure to a traumatic event that could then cause PTSD. Second, an abnormality may be an antecedent vulnerability factor for developing PTSD upon exposure to a traumatic event. Third, an abnormality may be the consequence of exposure to the traumatic event alone, in which case it would be found in both PTSD and non-PTSD trauma survivors equally but not in unexposed persons. Fourth, the abnormality may be a manifestation or product of the PTSD, that is, a PTSD sign. Fifth, the abnormality may be the product of a sequel or complication of PTSD.

\section{Difficulties with Prospective Studies}

The most scientific way to resolve competing explanations of the origin of a biological abnormality in PTSD is to measure the biological factor in individuals prior to their experiencing a traumatic event and then again afterward. Such prospective research, however, is made difficult and expensive by the considerations that a percentage of persons initially measured will not experience a traumatic event during the study period, a percentage of persons who do experience a traumatic event will fail to develop PTSD, and a percentage of those who do develop PTSD will be lost to follow-up. If these percentages are large, as they are likely to be, a prohibitive number of subjects may need to be measured before the traumatic event in order to obtain a sufficient number of suitable PTSD subjects after the traumatic event has occurred to have sufficient power for statistical analysis.

\section{Use of Twins as an Alternative to Prospective Studies}

A creative alternative to the prospective design is to identify surrogates for what the traumaexposed person would be like but for the experience of the traumatic event. The best such surrogate is a nontrauma-exposed, identical twin, who shares all the genes of the exposed twin and much of the exposed twin's early developmental environment (e.g., same family, same community, same school). In contrast to "shared" environment, "unique" environment designates environmental factors that one twin does not have in common with the other, for example, the traumatic exposure. A limitation of a design that incorporates only identical (monozygotic or MZ) twins is that it is incapable of teasing apart the contributions of heredity and shared environment, which must be lumped under the term familial. To distinguish these elements requires data from fraternal (dizygotic) twins, who putatively have the same shared environment as their cotwin but only half their alleles. Unfortunately, the work reported herein has not included fraternal twins for the reasons of expense and limited power (twice as many fraternal twin subjects as identical twin subjects are needed).

\section{Hypothetical Origins of a Biological Abnormality}

Figure $1 \mathrm{~A}$ to $\mathrm{F}$ illustrates for each of the hypothetical origins of a biological abnormality found in PTSD described above the data pattern that would be expected in an identical cotwin, casecontrol design. (The figures assume that the abnormality confers a higher value in persons with PTSD compared to persons without PTSD; if the opposite were the case, the patterns would be inverse). 


\section{Use of Twins in Biological PTSD Research}

We have used an identical cotwin, case-control design to resolve the competing explanations of the origin of some biological abnormalities (or "markers") found in PTSD. Investigating these abnormalities has advanced our understanding of PTSD, because they provide objective measures of the disorder that go beyond self-reported symptoms. They also have the potential for informing PTSD's pathogenesis. Unfortunately, this approach is unsuitable for the study of abnormal responses to trauma-related stimuli, because such stimuli would not be salient for the nontrauma-exposed cotwins. However, a number of biological abnormalities that do not involve responses to trauma-related stimuli have been found in PTSD. In published studies we have measured the biological markers described below in identical twins discordant for combat exposure in Vietnam with the goal of clarifying their origin.

\section{METHODS}

Our overall sample consists of 130 male Mz twin pairs, in which one twin served in combat in Vietnam and his cotwin did not. Zygosity was determined by a questionnaire that has been found $97 \%$ accurate when validated against blood typing. ${ }^{2}$ The only exclusion criterion for participation in these noninvasive studies was a serious contraindicating medical (e.g., cardiac) or psychiatric (e.g., psychosis, dementia) condition.

Most the twin pairs were recruited from the Vietnam Era Twin (VET) Registry, which has been described in detail elsewhere. ${ }^{3}$ VET Registry staff identified a pool of 624 living mz pairs of Vietnam era veterans discordant for combat exposure in Vietnam with known addresses and/ or telephone numbers. They succeeded in obtaining preliminary data over the telephone in both twins in 442 (71\%) of these pairs. These data included the Mississippi Scale for CombatRelated PTSD in the combat-exposed twin. ${ }^{4}$ VET Registry staff initially sent letters to each member of twin pairs randomly selected from the subpool of 442 pairs, inviting him to participate in the study. The letter contained a brief description of the study and copies of the consent documents the subject would be required to sign prior to participation. The letters were followed by telephone contact to answer any questions the subject candidate might have and to encourage participation. Unwillingness of one twin disqualified the pair. When recruitment of PTSD twin pairs (i.e., pairs in which the combat-exposed twin had PTSD) fell behind that of non-PTSD twin pairs, random recruitment was abandoned, and efforts were focused on the combat-exposed subject candidates who had the highest Mississippi scale scores or other suggestive evidence of PTSD from past mail and telephone surveys. Of 210 (48\% of the 442) VET Registry twin pairs invited to come to the Manchester, NH VA Research Service Psychophysiology Laboratory, 103 (49\%) participated. Most subject candidates who did not participate either declined because they were uninterested, too busy, or could not be scheduled at a time of mutual convenience.

After the VET Registry resource had apparently been exhausted for suitable PTSD pair candidates, to make up the deficit, letters were sent to approximately 80,000 Vietnam veterans receiving compensation for PTSD from the Veterans Benefits Administration. The letter inquired whether the recipient had an identical twin who had not served in combat in Vietnam, and if so, whether he and his brother were interested in participating in a research study. Twenty-seven additional PTSD pairs were recruited in this manner.

Due to ambiguous predictions for veterans with past but not current PTSD twin, some of whom may have substantial residual PTSD abnormalities, and others of whom may not, data from 27 past-PTSD (in the combat-exposed twin) twin pairs were excluded from these studies, leaving 50 PTSD and 53 non (never had)-PTSD twin pairs. All these subjects participated in an initial psychophysiological study. ${ }^{5}$ Subsequently, a convenience sample of 25 PTSD and 24 non- 
PTSD pairs from this larger sample returned for neuroimaging. For technical and other reasons described in the respective publications, not all of these subjects' data were usable in all studies.

All studies were approved by the Manchester, NH VA Medical Center Institutional Review Board. After the procedures had been fully explained, subjects gave written informed consent for participation.

The PTSD status of each combat-exposed subject was determined by the Clinician-

Administered PTSD Scale (CAPS) administered with regard to his combat experience. Further methodological details are available in the respective publications cited below.

The general statistical analytic approach to the data entailed a mixed model that treated PTSD Diagnosis (i.e., PTSD versus non-PTSD in the combat-exposed twin) as a between-pairs fixed effect, combat exposure as a within-pair fixed effect, and twin pairs as a random effect. ${ }^{6}$ The combat-unexposed cotwins of combat veterans with PTSD are designed "high-risk/ vulnerability" because their twin, with whom they share the same genes and developmental environment, developed PTSD. The combat-unexposed cotwins of combat veterans without PTSD are designed "low-risk/vulnerability" for the inverse reason.

\section{RESULTS}

\section{Heart Rate Response (HRR) to Loud Tones}

Larger HRRs to sudden, loud (startling) tones represent one of the best replicated psychophysiological markers for PTSD (reviewed in Ref. 7). Although the neuroanatomy of the HRR to startling stimuli is not as clearly delineated as the muscular response, the amygdala, the key brain structure for fear, likely plays a crucial role. In rodents, amygdala neurons are preferentially activated by high-intensity acoustic stimuli. ${ }^{8}$ Amygdalectomized monkeys are impaired in their HRRs to tones. ${ }^{9}$ Lesions of the central nucleus of the amygdala have been found to significantly reduce HRRs to startling white noise in rats, a finding that led investigators to conclude that the amygdala mediates responses to nonsignal acoustic stimuli, and that acceleratory HRRs can reflect the development of fear during acoustic startle testing. 10

There are at least two plausible explanations of increased amygdala reactivity in PTSD. Persons who generally react with greater fear to begin with are likely to have a more intense emotional reaction to traumatic events, which would be more likely to lead to the development of PTSD. Alternately, persons whose amygdalae are extremely activated by a terrifying traumatic event to the extent that they develop PTSD may have their amygdalae sensitized to further events. We attempted to resolve these competing explanations of the origins of increased HRRs to loud tones in our twin sample.

We presented a series of 15 95-dB (SPL), 1000-Hz, 500-ms pure tones with 0 -ms rise and fall times to our twin subjects and measured their HRRs. ${ }^{5}$ Stimuli of this intensity and configuration typically elicit a startle response upon initial presentation. Group mean HRRs are shown in Figure $2 \mathrm{~A}$. The analysis revealed a significant Diagnosis $\times$ Exposure interaction, with the combat-exposed twins with PTSD showing larger HRRs than their own identical, combatunexposed cotwins and than the combat-exposed twins without PTSD and their twins.

These findings suggest that increased HRRs to sudden, loud tones cannot be attributed to either heredity or familial environment shared by a PTSD combat veteran and his noncombatexposed, identical twin brother. Rather, it must reflect the contribution of unique environmental difference(s) between these twins. A limitation of the design employed here is that it cannot identify the specific unique environmental difference(s) between the combat-exposed and 
unexposed twins that is responsible for the abnormality. However, because the most salient, common difference was the presence of combat-related PTSD in the twins with the higher HRRs, and because this effect remained significant after considering the contributions of numerous potentially confounding variables, ${ }^{5}$ it is reasonable to attribute the larger HRRs to the presence of combat-related PTSD. In other words, increased HRRs to sudden, loud tones appear to be an acquired PTSD sign. The design employed here cannot rule out the possibility that the noncombat-exposed cotwins of combat veterans with PTSD share latent inherited phenotypes predisposing to increased HRRs that require traumatic exposure in order to be activated.

It is currently believed that hyper(re)activity of the amygdala underlies a number of PTSD symptoms. The results of the present study suggest that amygdale hyper(re)activity is not present prior to the occurrence of the traumatic event but rather develops following its occurrence in persons who develop PTSD. This postulate is worth testing within a twin design that employs more direct measures of amygdala (re)activity. ${ }^{11}$

\section{Neurological Soft Signs (NSSs)}

In previous work with nontwins, we found that male combat veterans, ${ }^{12,13}$ as well as adult females sexually abused as children ${ }^{13}$ with chronic PTSD are more neurologically compromised than comparably exposed persons without PTSD, as evidenced by more NSSs. These are various subtle indices of neurological dysfunction that are distinct from hard signs because they are difficult to localize to a specific brain region.

In our twin study, a neurologist/psychiatrist examined 45 NSSs and scored each on a 0-3 ordinal scale with specific anchors for each item. ${ }^{14}$ Scores were averaged prior to analysis. Group mean average NSS scores are shown in Figure 2 B. There was a significant Diagnosis main effect in the absence of a significant Exposure main effect or Diagnosis $\times$ Exposure interaction. PTSD combat veterans had significantly higher average NSS scores than nonPTSD combat veterans. The unexposed (high-risk) cotwins of the former also had significantly average higher NSS scores than the (low-risk) unexposed cotwins of the latter.

These results support the conclusion that subtle neurological dysfunction in PTSD is not acquired along with the PTSD, but rather represents a familial risk or vulnerability factor, which likely antedates the traumatic exposure. Especially supporting the premorbid interpretation is the finding that the high-risk unexposed cotwins had significantly higher average NSS scores than the low-risk unexposed cotwins. The finding that average NSS score in the unexposed cotwins was not associated with combat severity in their combat-exposed twin brothers refutes the interpretation that subtle neurological dysfunction in this study increased the risk of PTSD by increasing the likelihood of exposure to more severe combat events. Rather, preexisting subtle neurological dysfunction appears to confer vulnerability to developing PTSD following the occurrence of a psychologically traumatic event.

The increased NSSs found in this study point to subtle cortical dysfunction in PTSD combat veterans and their combat-unexposed twins. Failure of cortical inhibitory control over conditioned emotional responses may play a role in the pathogenesis of PTSD. The presence of antecedent, subtle neurological dysfunction may also make it less likely that patients with PTSD will recover, and thereby predispose them to a chronic course. It is also possible to state these relationships inversely. That is, a healthy nervous system may confer resilience in the face of highly stressful life events. 


\section{Hippocampal Volume}

Several structural magnetic resonance imaging (MRI) studies that employed anatomic segmentation have found lower gray matter volumes in the hippocampus in PTSD (e.g., see Refs. $15^{-17}$ ). In animals, exposure to severe and chronic stress has been shown to damage the hippocampus. ${ }^{18,19}$ It has been hypothesized that such damage may provide a basis for hipppocampal diminution in PTSD. ${ }^{20}$ Controversy exists, however, over the nature and origin of reduced hippocampal volume in this disorder. ${ }^{21-25}$ In order to address this issue, we performed a structural MRI study that manually traced the outlines of hippocampal gray matter in a subset members of our twin sample. ${ }^{26}$ The results were more revealing when the PTSD pairs were restricted to those in which the combat veteran had a Mississippi PTSD Scale score $>65$, indicative of more severe PTSD.

Group mean hippocampal volumes are shown in Figure $2 \mathrm{C}$. There was a highly significant Diagnosis main effect in the absence of an Exposure main effect or interaction. Hippocampal volumes were smaller in both the exposed and unexposed members of twin pairs in which the combat-exposed brother developed more severe PTSD, but no differences were observed in hippocampal volume between exposed and unexposed members of twin pairs regardless of PTSD status. These results provide evidence that smaller hippocampi in PTSD represent either a familial risk factor for trauma exposure or a familial vulnerability factor for the development of PTSD upon traumatic exposure. Because combat severity was not significantly related to hippocampal volume in this study, the latter explanation is favored. The lack of a significant combat exposure effect or diagnosis by exposure interaction argues against a stress-induced neurotoxicity hypothesis.

\section{Abnormal Cavum Septum Pellucidum (CSP)}

CSP is a space, or cavum, between two thin translucent leaflet membranes in the brain composed of white matter and surrounded by the gray matter of the septum pellucidum. Incomplete fusion results in the persistence of CSP, which may reflect incomplete neurodevelopment. A small degree of CSP appears to be a normal variant. However, an abnormally large CSP occurs more frequently in schizophrenics. ${ }^{27}$ One study reported the presence of CSP in 50\% of Israeli combat veterans with PTSD, compared to 14\% in normal volunteers matched for age, socioeconomic background, and military experience. The authors concluded that CSP may be an antecedent marker for psychopathological vulnerability to stress. ${ }^{28}$

We examined the presence of abnormal CSPs in the MRI scans of the same twin subjects in whom we measured hippocampal volume. Percentages of subjects in the various groups who showed abnormally large CSPs appear in Figure 2 D. As with NSSs and hippocampal volume, the results revealed a PTSD Diagnosis main effect in the absence of a Combat Exposure main effect or an interaction. These results also support the conclusion that the presence of an abnormal CSP is a familial risk/vulnerability factor for PTSD. However, there was no significant association between CSP and hippocampal volume. ${ }^{29}$

\section{CONCLUSIONS}

Up to the time of this writing, we have published results from our sample of identical twins discordant for combat exposure for four biologic markers for PTSD: HRRs to sudden, loud (startling) tones, NSSs indicative of subtle neurological dysfunction, volume of hippocampus, and the presence of abnormal CSP. Further studies of brain event-related potentials, HPA axis measures, structural MRI voxel-based morphometry, and functional MRI responses of amygdala, anterior cingulate cortex, and hippocampus are in progress. 
Assuming that a combat veteran's identical twin is a valid representation of what the combat veteran would have been like but for the experience of combat, the results with the four markers studied to date summarized herein suggest two major conclusions. First, as is recognized clinically for emotional responses, physiological responses may be altered as the result of being exposed to a severe traumatic event and developing PTSD. Because physiological responses must have an underlying brain basis, this implies that neuronal plasticity plays a role in the PTSD's pathogenesis.

Second, not all brain functional or structural differences that are observed in PTSD reflect trauma-induced plasticity. Subtle impairment of neurological dysfunction, as manifest in increased NSSs, appears to represent a familial, probably preexisting, vulnerability factor for the development of PTSD upon exposure to an extreme, psychologically traumatic event. Familial structural counterparts of neurological dysfunction include smaller hippocampi and incomplete brain development as manifest in the presence of abnormal CSP.

It has become a simple fact that behavioral phenotypes, including mental disorders, are the products of interactions between genes and environment. There is no doubt that this applies to PTSD, which represents the clearest example among mental disorders of the psychopathological effects of environmental event(s) yet has also been estimated to be 13$34 \%$ genetically determined. ${ }^{30}$ Ascertaining which genetic and environmental factors are involved in the development of PTSD, and how they interact, however, is anything but simple and will require a great deal of research to accomplish. The study of identical twins discordant for trauma exposure offers one means toward this end.

\section{Acknowledgments}

Additional Harvard/VA PTSD Twin Study investigators include Seth A. Eisen, Gregory M. Gillette, Jack Goldberg, William G. Henderson, Terence M. Keane, Michael J. Lyons, Alexander C. McFarlane, Stephen R. Paige (deceased), Arieh Y. Shalev, William R. True, Ming T. Tsuang, and Frank W. Weathers. The authors thank M. Macklin and H. Croteau for assistance. This work was supported by USPHS Grant R01MH54636 to Dr. Pitman; a U.S. Department of Veterans Affairs Merit Review Grant to Dr. Gilbertson; and USPHS Grant K02MH01110 to Dr. Shenton. The U.S. Department of Veterans Affairs also provided financial support for the development and maintenance of the Vietnam Era Twin (VET) Registry. Through their support of the VET Registry, numerous other U.S. organizations also provided invaluable assistance, including: Department of Defense; National Personnel Records Center; National Archives and Records Administration; Internal Revenue Service; National Institutes of Health; National Opinion Research Center; National Research Council; National Academy of Sciences; and Institute for Survey Research, Temple University. The authors gratefully acknowledge the continued cooperation and participation of the members of the VET Registry and their families, without whose contribution this research would not have been possible.

\section{REFERENCES}

1. American Psychiatric Association. Diagnostic and Statistical Manual of Mental Disorders. Washington, DC: American Psychiatric Press; 1994.

2. Eisen S, Neuman R, Goldberg J, et al. Determining zygosity in the Vietnam Era Twin Registry: an approach using questionnaires. Clin. Genet 1989;35:423-432. [PubMed: 2736790]

3. Henderson WG, Eisen S, Goldberg J, et al. The Vietnam Era Twin Registry: a resource for medical research. Public Health Rep 1990;105:368-373. [PubMed: 2116638]

4. Keane TM, Caddell JM, Taylor KL. Mississippi Scale for Combat-Related Posttraumatic Stress Disorder: three studies in reliability and validity. J. Consult. Clin. Psychol 1988;56:85-90. [PubMed: 3346454]

5. Orr SP, Metzger LJ, Lasko NB, et al. Physiologic responses to sudden, loud tones in monozygotic twins discordant for combat exposure: association with posttraumatic stress disorder. Arch. Gen. Psychiatry 2003;60:283-288. [PubMed: 12622661]

6. Little, RC.; Milliken, GA.; Stroup, WW.; Wolfinger, RD. SAS System for Mixed Models. Cary, NC: SAS Institute; 1996. 
7. Orr SP, Metzger LJ, Pitman RK. Psychophysiology of post-traumatic stress disorder. Psychiatr. Clin. North Am 2002;25:271-293. [PubMed: 12136501]

8. Bordi F, Ledoux J. Sensory tuning beyond the sensory system: an initial analysis of auditory response properties of neurons in the lateral amygdaloid nucleus and overlying areas of the striatum. J. Neurosci 1992;12:2493-2503. [PubMed: 1613543]

9. Bagshaw MH, Benzies S. Multiple measures of the orienting reaction and their dissociation after amygdalectomy in monkeys. Exp. Neurol 1968;20:175-187. [PubMed: 4968336]

10. Young BJ, Leaton RN. Amygdala central nucleus lesions attenuate acoustic startle stimulus-evoked heart rate changes in rats. Behav. Neurosci 1996;110:228-237. [PubMed: 8731050]

11. Rauch SL, Whalen PJ, Shin LM, et al. Exaggerated amygdala response to masked facial stimuli in posttraumatic stress disorder: a functional MRI study. Biol. Psychiatry 2000;47:769-776. [PubMed: 10812035]

12. Gurvits TV, Lasko NB, Schachter SC, et al. Neurological status of Vietnam veterans with chronic posttraumatic stress disorder. J. Neuropsychiatry Clin. Neurosci 1993;5:183-188. [PubMed: 8508036]

13. Gurvits TV, Gilbertson MW, Lasko NB, et al. Neurologic soft signs in chronic posttraumatic stress disorder. Arch. Gen. Psychiatry 2000;57:181-186. [PubMed: 10665621]

14. Gurvits TV, Metzger LJ, Lasko NB, et al. Subtle neurological compromise as a vulnerability factor for combat-related post-traumatic stress disorder: results of a twin study. Arch. Gen. Psychiatry 2006;63:571-576. [PubMed: 16651514]

15. Bremner JD, Randall P, Scott TM, et al. MRI-based measurement of hippocampal volume in patients with combat-related posttraumatic stress disorder. Am. J. Psychiatry 1995;152:973-981. [PubMed: 7793467]

16. Gurvits TV, Shenton ME, Hokama H, et al. Magnetic resonance imaging study of hippocampal volume in chronic, combat-related posttraumatic stress disorder. Biol. Psychiatry 1996;40:10911099. [PubMed: 8931911]

17. Villarreal G, Hamilton DA, Petropoulos H, et al. Reduced hippocampal volume and total white matter volume in posttraumatic stress disorder. Biol. Psychiatry 2002;52:119-125. [PubMed: 12114003]

18. Sapolsky RM, Uno H, Rebert CS, Finch CE. Hippocampal damage associated with prolonged glucocorticoid exposure in primates. J. Neurosci 1990;10:2897-2902. [PubMed: 2398367]

19. McEwen BS. The neurobiology of stress: from serendipity to clinical relevance. Brain Res 2000;886:172-189. [PubMed: 11119695]

20. Sapolsky RM. Why stress is bad for your brain. Science 1996;273:749-750. [PubMed: 8701325]

21. Pitman RK. Hippocampal diminution in PTSD: more (or less?) than meets the eye. Hippocampus 2001;11:73-74. [PubMed: 11345126]

22. Bremner JD. Hypotheses and controversies related to effects of stress on the hippocampus: an argument for stress-induced damage to the hippocampus in patients with posttraumatic stress disorder. Hippocampus 2001;11:75-81. [PubMed: 11345127]

23. McEwen BS. Commentary on PTSD discussion. Hippocampus 2001;11:82-84.

24. Yehuda R. Are glucocortoids responsible for putative hippocampal damage in PTSD? How and when to decide. Hippocampus 2001;11:85-89. [PubMed: 11345128]

25. Sapolsky RM. Atrophy of the hippocampus in posttraumatic stress disorder: how and when? Hippocampus 2001;11:90-91. [PubMed: 11345129]

26. Gilbertson MW, Shenton ME, Ciszewski A, et al. Smaller hippocampal volume predicts pathologic vulnerability to psychological trauma. Nat. Neurosci 2002;5:1242-1247. [PubMed: 12379862]

27. Kasai K, McCarley RW, Salisbury DF, et al. Cavum septi pellucidi in first-episode schizophrenia and first-episode affective psychosis: an MRI study. Schizophr. Res 2004;71:65-76. [PubMed: 15374574]

28. Myslobodsky MS, Glicksohn J, Singer J, et al. Changes of brain anatomy in patients with posttraumatic stress disorder: a pilot magnetic resonance imaging study. Psychiatry Res 1995;58:259-264. [PubMed: 8570781]

29. May FS, Chen QC, Gilbertson MW, et al. Cavum septum pellucidum in monozygotic twins discordant for combat exposure: relationship to PTSD. Biol. Psychiatry 2004;55:656-658. [PubMed: 15013837] 
30. True WR, Rice J, Eisen SA, et al. A twin study of genetic and environmental contributions to liability for posttraumatic stress symptoms. Arch. Gen. Psychiatry 1993;50:257-264. [PubMed: 8466386] 
(A)

血 ExP+Combat-Exposed Twin with PTSD

[ ExP- Combat-Exposed Twin without PTSD

豆 $\mathbf{U X P}+$ High-risk/vulnerability unexposed co-twin of ExP+

目 UxP- Low-risk/vulnerability unexposed co-twin of ExP-

\section{FACTORS:}

Diagnosis (between-pairs): Combat-related PTSD diagnosis in the Ex twin : P+ (heavy bars ), P- (light bars)

Exposure (within-pair): Combat-Exposed (vertical shading), Unexposed (horizontal shading)

\section{(C) Consequence of Exposure} (independent of PTSD)

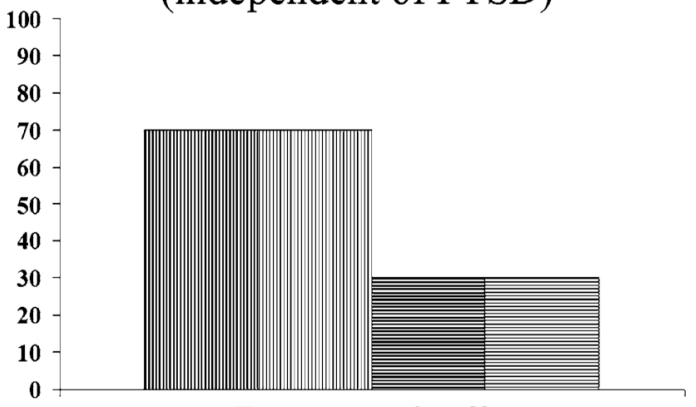

Exposure main effect t-test: ExP- > UxP-

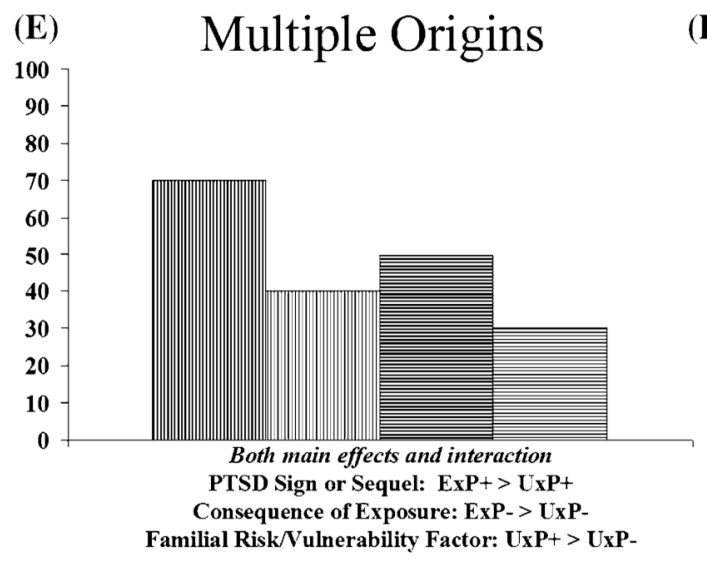

(B)

PTSD Sign or Sequel

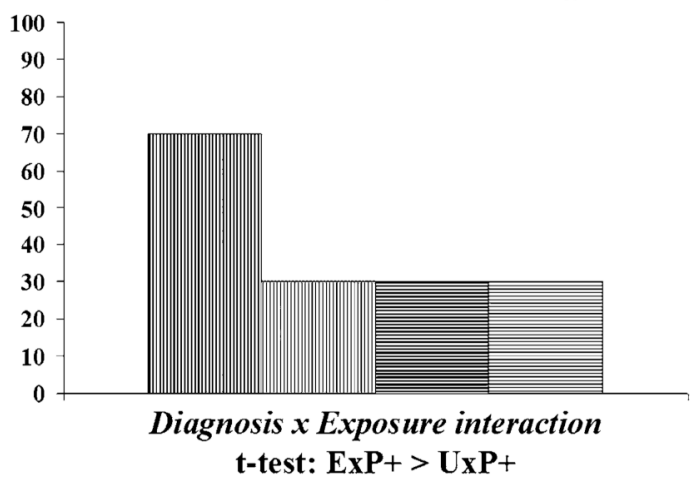

(D)

\section{Familial Risk or Vulnerability Factor}

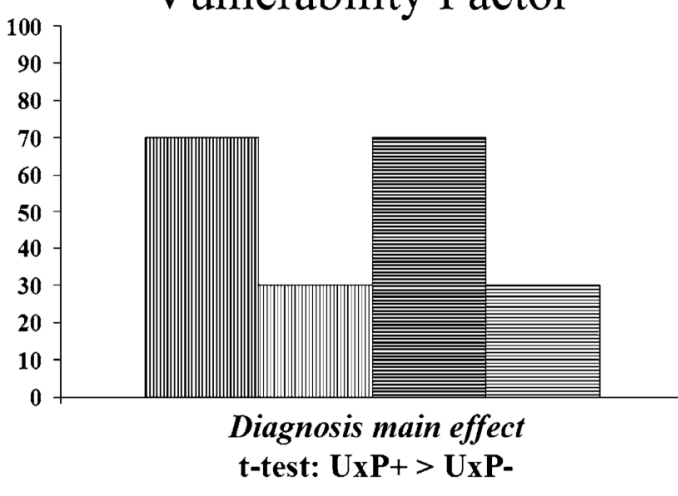

(F) Non-Replication of Abnormality

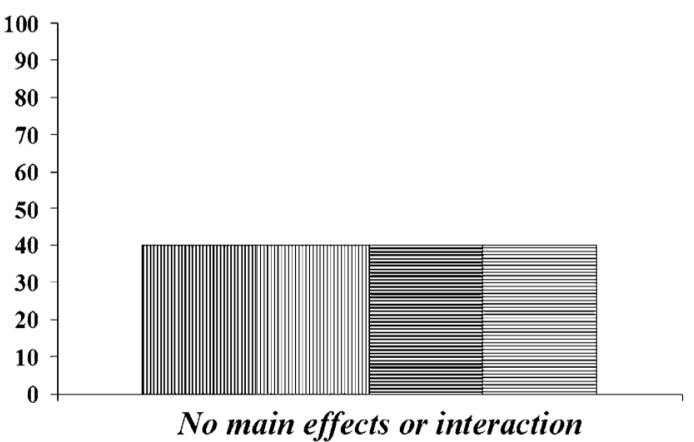

No main effects or interaction

FIGURE 1.

Predicted data patterns for various hypothetical origins of a biological abnormality in PTSD. 

(A) Heart Rate Response to Loud Tones

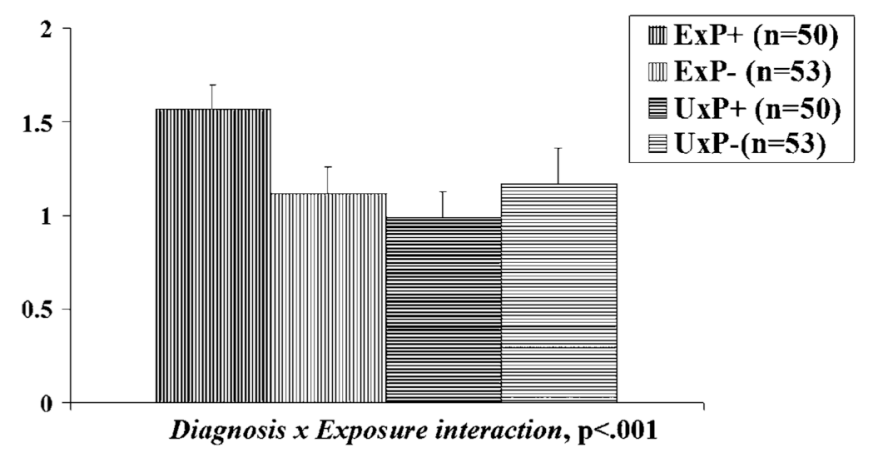

(C) Hippocampal Volume

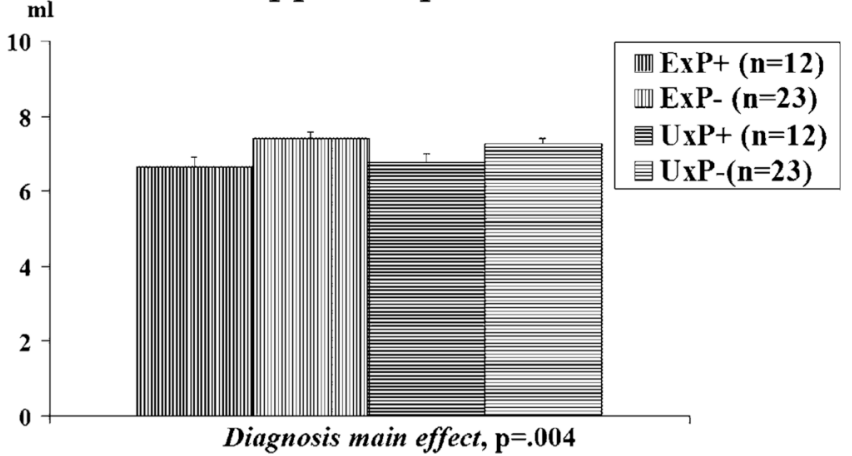

(B) Neurological Soft Sign Score

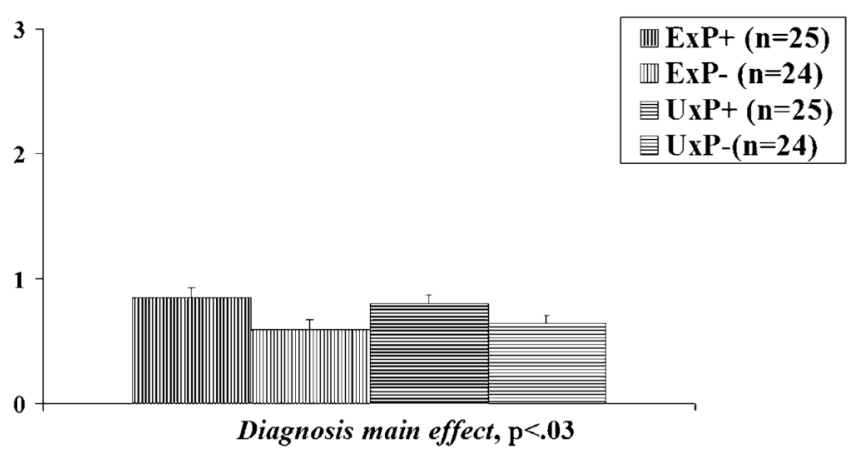

(D) Cavum Septum Pellucidum

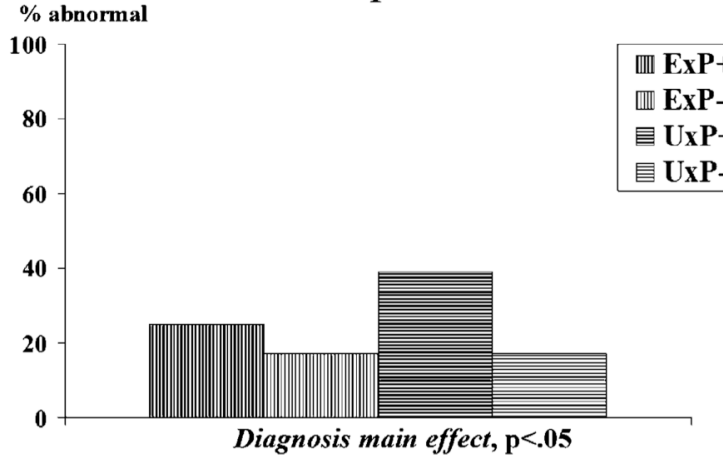

FIGURE 2.

Groups means for biological abnormalities. Error bars indicate SEM. 